\title{
Reduced basis approximation for shape optimization in thermal flows with a parametrized polynomial geometric map
}

\author{
Gianluigi Rozza, Toni Lassila, and Andrea Manzoni
}

\begin{abstract}
Reduced basis approximations for geometrically parametrized advection-diffusion equations are investigated. The parametric domains are assumed to be images of a reference domain through a piecewise polynomial map; this may lead to nonaffinely parametrized diffusion tensors that are treated with an empirical interpolation method. An a posteriori error bound including a correction term due to this approximation is given. Results concerning the applied methodology and the rigor of the corrected error estimator are shown for a shape optimization problem in a thermal flow.
\end{abstract}

\section{Introduction}

We consider the parametrized advection-diffusion equation in a bounded and piecewise smooth domain $\Omega_{o}(\boldsymbol{\mu}) \subset \mathbb{R}^{2}$, whose shape depends on a vector of geometrical parameters $\boldsymbol{\mu}$ residing in a low-dimensional parameter space $\mathcal{D} \subset \mathbb{R}^{P}$ (e.g. $P \leq 10$ ). The weak form of the equation reads as follows: for any given $\boldsymbol{\mu} \in \mathcal{D}$, find $u \in H^{1}\left(\Omega_{o}(\boldsymbol{\mu})\right)$ s.t. $u=u_{D}$ on $\Gamma_{D}$ and

$$
\int_{\Omega_{o}(\boldsymbol{\mu})}(\varepsilon \nabla u \cdot \nabla v+v \mathbf{b} \cdot \nabla u) d \Omega=\int_{\Omega_{o}(\boldsymbol{\mu})} f v d \Omega \quad \forall v \in H^{1}\left(\Omega_{o}(\boldsymbol{\mu})\right)
$$

where $\mathbf{b}$ is a given divergence-free constant vector field, $\Gamma_{D}$ denotes the Dirichlet boundary, while homogeneous Neumann conditions are imposed on $\Gamma_{N}=\partial \Omega \backslash \Gamma_{D}$. Our interest is to solve equation (1) in a way that is:

Gianluigi Rozza · Andrea Manzoni

MATHICSE-CMCS Modelling and Scientific Computing, École Polytechnique Fédérale de Lausanne, Switzerland, e-mail: gianluigi.rozza@epfl.ch, andrea.manzoni@epfl.ch

Toni Lassila

Institute of Mathematics, Helsinki University of Technology, Finland, e-mail: toni.lassila@tkk.fi 
- efficient in the sense that for any $\boldsymbol{\mu}$ a numerical solution is obtained in real-time for arbitrarily fine discretizations;

- reliable in the sense that for any $\boldsymbol{\mu}$ the obtained solution is verifiable within some prescribed tolerance from the finite element solution computed using a very fine mesh for discretization. Any error bound should be rigorous, that is to say, it should be a safe upper bound for the true error.

To that end, we employ the reduced basis (RB) method originally developed for nonlinear structural mechanics in the 1980s and more recently systematized for elliptic and parabolic, coercive and noncoercive, PDEs. The method is analyzed in detail in $[9,13]$ and in their references; previous works on reduced basis methods for the advection-diffusion equation include [3, 10, 15]. This method is a model reduction scheme for parametric PDEs based on the use of "snapshot" finite element solutions of the PDE (for certain values of the parameters) as global approximation basis functions. Our objective is to use the efficient evaluation of the RB solutions in a multi-query context, required for example in shape optimization of PDE modelled systems.

\section{Reduced basis approximation of parametric advection-diffusion equations}

We assume that the parametric domains $\Omega_{o}(\boldsymbol{\mu})$ are obtained by mapping from a reference domain $\Omega$ with $T(\boldsymbol{x}, \boldsymbol{\mu})$ a piecewise polynomial map w.r.t both arguments, as $\Omega \mapsto \Omega_{o}(\boldsymbol{\mu}):=T(\Omega, \boldsymbol{\mu})$. Problem (1) is thus traced back to the reference domain as

$$
\int_{\Omega}\left(\varepsilon \nu_{T} \nabla u \cdot \nabla v+v \chi_{T} \mathbf{b} \cdot \nabla u\right) d \Omega=\int_{\Omega} \eta_{T} f v d \Omega \quad \forall v \in \mathcal{X} \equiv H^{1}(\Omega),
$$

where the parametric transformation tensors $\nu_{T}(\mathbf{x}, \boldsymbol{\mu}), \chi_{T}(\mathbf{x}, \boldsymbol{\mu})$ and $\eta_{T}(\mathbf{x}, \boldsymbol{\mu})$ are obtained from a change of coordinates (with the Jacobian of $T$ denoted by $\left.J_{T}\right)$ as

$$
\nu_{T}=J_{T}^{-T} J_{T}^{-1}\left|J_{T}\right|, \quad \chi_{T}=J_{T}^{-1}\left|J_{T}\right|, \quad \eta_{T}=\left|J_{T}\right| .
$$

We may rewrite (2) as

$$
\mathcal{A}(u, v ; \boldsymbol{\mu})=f(v ; \boldsymbol{\mu}) \quad \forall v \in \mathcal{X},
$$

where the parametric bilinear form $\mathcal{A}(\cdot, \cdot ; \boldsymbol{\mu})$ is coercive and the linear functional $f(\cdot ; \boldsymbol{\mu})$ is continuous. The standard Galerkin finite element (FE) approximation of $(4)$ is to find $u^{\mathcal{N}} \in \mathcal{X}^{\mathcal{N}}$ s.t. $\mathcal{A}\left(u^{\mathcal{N}}, v ; \boldsymbol{\mu}\right)=f(v ; \boldsymbol{\mu})$ for all $v \in$ $\mathcal{X}^{\mathcal{N}}$, where $\mathcal{X}^{\mathcal{N}}$ is a FE space constructed by using e.g. piecewise linear shape functions on a discrete mesh [11]. Here we denote by $\mathcal{N}$ the dimension of the FE space, which is assumed to be large enough that the repeated assembly and solution of the FE system is too expensive for a multi-query context. 
In order to find an approximation to $u^{\mathcal{N}}$ in an efficient and reliable way, we use Galerkin projection on a reduced subspace of basis functions. Let $\boldsymbol{\mu}^{1}, \ldots, \boldsymbol{\mu}^{N}$ be a collection of parameters and define the reduced basis approximation space as $\mathcal{X}_{N}^{\mathcal{N}}:=\operatorname{span}\left\{u^{\mathcal{N}}\left(\boldsymbol{\mu}^{n}\right): n=1, \ldots, N\right\}$, where each $u^{\mathcal{N}}\left(\boldsymbol{\mu}^{n}\right) \in \mathcal{X}^{\mathcal{N}}$ is a FE solution for a given parameter value $\boldsymbol{\mu}^{n}$. The reduced basis formulation reads as follows: find $u_{N}^{\mathcal{N}} \in \mathcal{X}_{N}^{\mathcal{N}}$ s.t. $\mathcal{A}\left(u_{N}^{\mathcal{N}}, v ; \boldsymbol{\mu}\right)=f(v)$, for all $v \in \mathcal{X}_{N}^{\mathcal{N}}$. In practice, an orthonormalization procedure is required to build a basis $\left\{\Phi_{n}\right\}_{n=1}^{N}$ for the RB space $\mathcal{X}_{N}^{\mathcal{N}}$ that guarantees algebraic stability [9]. As long as the parametric bilinear form is affinely parametrized [13], that is to say of the form

$$
\mathcal{A}(u, v ; \boldsymbol{\mu})=\sum_{q=1}^{Q_{a}} \Theta_{a}^{q}(\boldsymbol{\mu}) a^{q}(u, v)+\sum_{q=1}^{Q_{b}} \Theta_{b}^{q}(\boldsymbol{\mu}) b^{q}(u, v)
$$

for some integers $Q_{a}, Q_{b}$, where $\Theta_{a}^{q}(\boldsymbol{\mu})=\beta_{k}^{i, j}(\boldsymbol{\mu}), \Theta_{b}^{q}(\boldsymbol{\mu})=\gamma_{k}^{i, j}(\boldsymbol{\mu}), q$ is a condexed index for $i, j, k$ and

$$
\begin{array}{cl}
a^{q(i, j, k)}(u, v)=\varepsilon \int_{\Omega} \xi_{k}^{i, j}(\mathbf{x}) \frac{\partial u}{\partial x_{i}} \frac{\partial v}{\partial x_{j}} d \Omega, & b^{q(i, j, k)}(u, v)=\int_{\Omega} \zeta_{k}^{i, j}(\mathbf{x}) b_{i} \frac{\partial u}{\partial x_{j}} v d \Omega, \\
\nu_{T}^{i, j}(\mathbf{x}, \boldsymbol{\mu})=\sum_{k=1}^{K_{i j}^{a}} \beta_{k}^{i, j}(\boldsymbol{\mu}) \xi_{k}^{i, j}(\mathbf{x}), & \chi_{T}^{i, j}(\mathbf{x}, \boldsymbol{\mu})=\sum_{k=1}^{K_{i j}^{b}} \gamma_{k}^{i, j}(\boldsymbol{\mu}) \zeta_{k}^{i, j}(\mathbf{x}),
\end{array}
$$

the solution of the reduced basis problem splits into two stages. In the socalled offline stage we assemble and store once and for all the parameterindependent system matrices $\underline{A}^{q}$ and $\underline{B}^{q}$ of components

$$
\left[\underline{A}^{q}\right]_{m, n}=a^{q}\left(\Phi_{n}, \Phi_{m}\right), \quad\left[\underline{B}^{q}\right]_{m, n}=b^{q}\left(\Phi_{m}, \Phi_{n}\right)
$$

using the global reduced basis functions $\Phi_{k}$, and similarly for the righthand-sides. Then in the online stage for a given parameter $\boldsymbol{\mu}$ the parametric coefficients $\Theta_{a}^{q}(\boldsymbol{\mu}), \Theta_{b}^{q}(\boldsymbol{\mu})$ are evaluated and the reduced basis matrix $\underline{\mathcal{A}}_{N}=\sum_{q=1}^{Q_{a}} \Theta_{a}^{q}(\boldsymbol{\mu}) \underline{A}^{q}+\sum_{q=1}^{Q_{b}} \Theta_{b}^{q}(\boldsymbol{\mu}) \underline{B}^{q}$ is assembled, and similarly for the right-hand-side. This linear system of dimension $N \times N$ is dense, but inexpensive to solve: the online complexity is independent of the FE solution dimension $\mathcal{N}$ and thus we fulfill the first requirement of efficiency.

The following greedy algorithm for choosing the parameters $\boldsymbol{\mu}^{n}$ has been used $[9,13,16]$. Let $\Xi_{\text {train }} \subset \mathcal{D}$ be a finite training sample of parameter points chosen according to a uniform or log-uniform distribution. Define the parameter-independent norm $\|v\|_{X}:=\sqrt{\mathcal{A}(v, v ; \overline{\boldsymbol{\mu}})+\lambda\|v\|_{L^{2}(\Omega)}}$ for some $\overline{\boldsymbol{\mu}} \in \mathcal{D}$ and $\lambda>0$ large enough such that the resulting norm is well-defined. Given the first parameter value $\boldsymbol{\mu}^{1}$ and a sharp, inexpensive a posteriori error bound $\Delta_{n}(\boldsymbol{\mu})$ for the norm $\|\cdot\|_{X}$ such that $\left\|u^{\mathcal{N}}(\boldsymbol{\mu})-u_{n}^{\mathcal{N}}(\boldsymbol{\mu})\right\|_{X} \leq \Delta_{n}(\boldsymbol{\mu})$ for all $\mu \in \Xi_{\text {train }}$, we choose the remaining parameter values as the solutions $\boldsymbol{\mu}^{n}=\arg \max _{\boldsymbol{\mu} \in \Xi_{\text {train }}} \Delta_{n-1}(\boldsymbol{\mu})$, for $n=2, \ldots, N$. The quality of the reduced basis approximation depends crucially on the quality of the a posteriori error estimator. The standard RB error estimator in literature [9] for problems 
that satisfy the affinity assumption (5) is

$$
\Delta_{N}(\boldsymbol{\mu}):=\frac{\|r(\cdot, \boldsymbol{\mu})\|_{X^{\prime}}}{\alpha_{\mathrm{LB}}(\boldsymbol{\mu})} \geq\left\|u_{N}^{\mathcal{N}}-u^{\mathcal{N}}\right\|_{X}=\|e(\boldsymbol{\mu})\|_{X},
$$

where $\|r(\cdot, \boldsymbol{\mu})\|_{X^{\prime}}$ is the dual norm of the residual $r(v, \boldsymbol{\mu})=f(v)-\mathcal{A}\left(u_{N}^{\mathcal{N}}, v ; \boldsymbol{\mu}\right)$ and $\alpha_{\mathrm{LB}}(\boldsymbol{\mu})$ is a computable lower bound for the discrete coercivity constant

$$
0<\alpha_{\mathrm{LB}}(\boldsymbol{\mu}) \leq \alpha(\boldsymbol{\mu})=\inf _{u \in \mathcal{X}^{\mathcal{N}}} \frac{\mathcal{A}(u, u ; \boldsymbol{\mu})}{\|u\|_{X}^{2}} .
$$

For efficient and reliable methods of computing both $\|r(\cdot, \boldsymbol{\mu})\|_{X^{\prime}}$ and $\alpha_{\mathrm{LB}}(\boldsymbol{\mu})$ we refer the reader to $[13,6,4,2]$. In the greedy basis construction algorithm we usually fix a priori an error tolerance $\varepsilon_{\text {tol }}^{R B}$ and then we continue the process until the condition $\Delta_{N}(\boldsymbol{\mu}) \leq \varepsilon_{\text {tol }}^{R B}$ for all $\boldsymbol{\mu} \in \Xi_{\text {train }}$ is achieved.

If the affinity assumption does not hold, we rely on the empirical interpolation method (EIM) [1], which is an interpolation method for parametric functions based on adaptively chosen interpolation points and global shape functions. When the geometric transformation $T(\mathbf{x}, \boldsymbol{\mu})$ is polynomial the advection tensor $\chi_{T}(\mathbf{x}, \boldsymbol{\mu})$ is polynomial and therefore always affinely parametrized, while the diffusion one $\nu_{T}(\mathbf{x}, \boldsymbol{\mu})$ is a nonaffine tensor. To approximate each component $\nu_{T}^{i, j}(\mathbf{x}, \boldsymbol{\mu})$ of the tensor we use a different set of interpolation points and thus look for an affine approximation

$$
\widetilde{\nu}_{T}^{i, j}(\mathbf{x}, \boldsymbol{\mu}):=\sum_{m=1}^{M_{i j}} \vartheta_{m}^{i, j}(\boldsymbol{\mu}) \xi_{m}^{i, j}(\boldsymbol{x})=\nu_{T}^{i, j}(\mathbf{x}, \boldsymbol{\mu})+\varepsilon^{i, j}(\boldsymbol{x} ; \boldsymbol{\mu}),
$$

with the error terms under some tolerance, i.e. $\left\|\varepsilon^{i, j}(\cdot ; \boldsymbol{\mu})\right\|_{\infty}<\varepsilon_{\text {tol }}^{\text {EIM }} \forall \boldsymbol{\mu} \in \mathcal{D}$.

For the reliability of the methodology we need to guarantee an a posteriori error bound between the "truth" finite element solution and the reduced basis approximation. The snapshot solutions $u^{\mathcal{N}}\left(\boldsymbol{\mu}^{n}\right)$ should be obtained by a FE stable method: for the advection-diffusion equation we can use a Galerkin formulation with either Galerkin least-squares (GLS) or streamline upwind (SUPG) stabilizers [11]. For more details on coupling the stabilizer with the reduced basis framework, see $[3,10]$. To simplify things we choose the physical Peclet number $\mathrm{Pe}=\varepsilon^{-1}$ small enough such that the finite element approximations are always guaranteed to be stable without adding any stabilizing terms. By applying the coercivity property it holds that

$$
\alpha(\boldsymbol{\mu})\|e(\boldsymbol{\mu})\|_{X}^{2} \leq \mathcal{A}(e(\boldsymbol{\mu}), e(\boldsymbol{\mu}) ; \boldsymbol{\mu}) .
$$

Using the ideas from $[1,8]$ we can prove an a posteriori error estimate of the form (7) also in the nonaffine case. Defining the trilinear forms $a_{i, j}(u, v, \varphi):=$ $\varepsilon \int_{\Omega} \varphi \frac{\partial u}{\partial x_{i}} \frac{\partial v}{\partial x_{j}} d \Omega$ and the residual of the reduced basis solution $u_{N}^{\mathcal{N}}$ as

$$
r_{N}(v ; \boldsymbol{\mu}):=f(v)-\sum_{q=1}^{Q_{b}} \Theta_{b}^{q}(\boldsymbol{\mu}) b^{q}\left(u_{N}^{\mathcal{N}}, v\right)-\sum_{i, j=1}^{2} a_{i, j}\left(u_{N}^{\mathcal{N}}, v, \widetilde{\nu}_{T}^{i, j}\right)
$$

we have the following a posteriori error bound: 


$$
\|e(\boldsymbol{\mu})\|_{X} \leq \frac{\left\|r_{N}(\cdot ; \boldsymbol{\mu})\right\|_{X^{\prime}}}{\alpha_{\mathrm{LB}}(\boldsymbol{\mu})}+\sup _{w \in \mathcal{X}} \frac{\sum_{i, j=1}^{2} a_{i, j}\left(u_{N}^{\mathcal{N}}, w, \nu_{T}^{i, j}-\widetilde{\nu}_{T}^{i, j}\right)}{\alpha_{\mathrm{LB}}(\boldsymbol{\mu})\|w\|_{X}} .
$$

By using the definitions of the error $e(\boldsymbol{\mu})$ and the residual we get

$$
\mathcal{A}(e(\boldsymbol{\mu}), e(\boldsymbol{\mu}) ; \boldsymbol{\mu}) \leq r_{N}(e(\boldsymbol{\mu}) ; \boldsymbol{\mu})-\sum_{i, j=1}^{2} a_{i, j}\left(u_{N}^{\mathcal{N}}(\boldsymbol{\mu}), e(\boldsymbol{\mu}), \nu_{T}^{i, j}-\widetilde{\nu}_{T}^{i, j}\right) ;
$$

by property (10) it follows that

$$
\|e(\boldsymbol{\mu})\|_{X} \leq \frac{1}{\alpha(\boldsymbol{\mu})}\left(\frac{r_{N}(e(\boldsymbol{\mu}) ; \boldsymbol{\mu})}{\|e(\boldsymbol{\mu})\|_{X}}-\frac{\sum_{i, j=1}^{2} a_{i, j}\left(u_{N}^{\mathcal{N}}, e(\boldsymbol{\mu}), \nu_{T}^{i, j}-\widetilde{\nu}_{T}^{i, j}\right)}{\|e(\boldsymbol{\mu})\|_{X}}\right)
$$

and (11) is obtained by taking sups. The correction term originating from the empirical interpolation is of order $O\left(\left|u_{N}^{\mathcal{N}}\right|_{1}\right)$ and therefore does not vanish as $N \rightarrow \infty$ if the number of terms in the empirical interpolation approximation is kept fixed. We need to choose the tolerance $\varepsilon_{\text {tol }}^{\text {EIM }} \ll \| r_{N}(\cdot ; \boldsymbol{\mu})||_{X^{\prime}} /\left(\varepsilon\left|u_{N}^{\mathcal{N}}\right|_{1}\right)$, so that the correction term does not dominate the error estimate. To obtain an error estimate computable online without $\mathcal{N}$-dependence we use the estimator $\Delta_{N}^{c o r r}(\boldsymbol{\mu})$ proposed in [1]:

$$
\|e(\boldsymbol{\mu})\|_{X} \lesssim \frac{\left\|r_{N}(\cdot ; \boldsymbol{\mu})\right\|_{X^{\prime}}}{\alpha_{\mathrm{LB}}(\boldsymbol{\mu})}+\sup _{w \in \mathcal{X}} \frac{\sum_{i, j=1}^{2} \widetilde{\varepsilon}_{M_{i j}}^{i, j} a_{i, j}\left(u_{N}^{\mathcal{N}}, w, \xi_{M_{i j}+1}^{i, j}\right)}{\alpha_{\mathrm{LB}}(\boldsymbol{\mu})\|w\|_{X}},
$$

where $\widetilde{\varepsilon}_{M_{i j}}^{i, j}:=\left|\nu_{T}^{i, j}\left(\boldsymbol{z}^{M_{i j}+1}, \boldsymbol{\mu}\right)-\widetilde{\nu}_{T}^{i, j}\left(\boldsymbol{z}^{M_{i j}+1}, \boldsymbol{\mu}\right)\right|$ is a one-point estimate for the error $\left\|\varepsilon^{i, j}(\cdot, \boldsymbol{\mu})\right\|_{L^{\infty}(\Omega)}$ computed using the $\left(M_{i j}+1\right)^{t h}$ interpolation point $\boldsymbol{z}^{M_{i j}+1}$. By "¿" we mean that the bound is no longer fully rigorous.

\section{Numerical example}

We consider an optimal heat exchange problem. A NACA0012 airfoil is placed in a thermal flow; our control variables are the vertical position of the airfoil and its shape (for small perturbations). The reference geometry is shown in Fig. 1. The objective is to obtain the correct desired average temperature $\bar{u}_{\text {target }}$ at the outflow given a fixed angle of attack $\sigma_{0}$ for the airfoil:

$$
\begin{array}{ll}
\min _{\boldsymbol{\mu} \in \mathcal{D}} & {\left[\bar{u}_{\text {target }}-\frac{1}{\left|\Gamma_{\text {out }}\right|} \int_{\Gamma_{\text {out }}} u(\boldsymbol{x}) d \Gamma\right]^{2}+\lambda\left[\sigma(\boldsymbol{\mu})-\sigma_{0}\right]^{2},} \\
\text { s.t. } & \int_{\Omega_{o}(\boldsymbol{\mu})}(\varepsilon \nabla u \cdot \nabla v+v \mathbf{b} \cdot \nabla u) d \Omega_{o}=\int_{\Omega_{o}(\boldsymbol{\mu})} f v d \Omega_{o} \quad \forall v \in H^{1}\left(\Omega_{o}(\boldsymbol{\mu})\right)
\end{array}
$$

with $u=T_{0}$ on $\Gamma_{\text {in }} \cup \Gamma_{\text {free }}, u=T_{1}$ on $\Gamma_{\text {surf }}, \quad u=T_{2}$ on the airfoil. 
We parametrize the geometry around the airfoil using free-form deformations (FFD) [14]: a $6 \times 6$ lattice of control points is placed around the airfoil and the closest four control points are allowed to move in the $x_{2}$-direction. This results in a polynomial geometric map $T(\mathbf{x}, \boldsymbol{\mu})$ with $P=4$ parameters built using Bernstein polynomials. In Fig. 1 we also display the control points and the deformation of the reference shape as the control points are moved. For more details on the FFD parametrization setup we refer the reader to [7]. For the finite element computations $\mathcal{N}=15,718$ degrees of freedom are used.
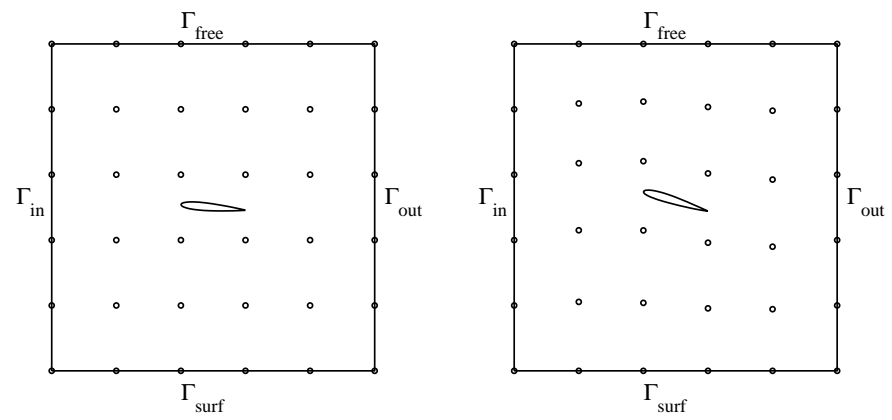

Fig. 1 Reference domain $\Omega$ and a deformed configuration $\Omega_{o}(\boldsymbol{\mu})$ using FFDs.

To solve the optimization problem (13), the algorithm based on sequential quadratic programming (SQP) provided in Matlab has been used; convergence to the optimal solution has been reached after 25 functional evaluations. In order to evaluate effectively the state equation in the constraint of (13) we replace the FE solution $u^{\mathcal{N}}$ with the RB approximation $u_{N}^{\mathcal{N}}$. The nonaffinely parametrized diffusion tensor has been approximated with the empirical interpolation method using a tolerance of $\varepsilon_{\text {tol }}^{\text {EIM }}=10^{-4}$ and, then, $\varepsilon_{\text {tol }}^{\text {EIM }}=10^{-6}$, resulting in $M=\sum_{i, j=1}^{2} M_{i j}=108$ and $M=208$ terms, respectively, in the affine expansion. After this the reduced basis offline stage consists of assembly of the matrices (6), performing the successive constraint method [6] for estimation of the lower bound $\alpha_{L B}(\boldsymbol{\mu})$ of the coercivity constant, and finally a greedy procedure for choosing the reduced basis snapshots and the corresponding basis functions. The maximum number of basis functions used was $N=38$. In Fig. 2 we show the error estimates (with and without the correction term from the empirical interpolation) $\Delta_{N}(\boldsymbol{\mu})$ and $\Delta_{N}^{\text {corr }}(\boldsymbol{\mu})$ as functions of $N$, compared to the true error $e(\boldsymbol{\mu})$ in the worst-case, for $M=108$ and $M=208$. In the first case the approximation performed by EIM is too poor and the correction term wider than in the second test, with a more accurate and rigorous error estimator. Moreover, in the first test we still have some "plateau" effect to be reduced [12]. For $M=208$ we observe a reduction of 140:1 in the time to solve the RB system vs. the assembling and solution of the FE system, while the reduction in the linear system size is 400:1. 

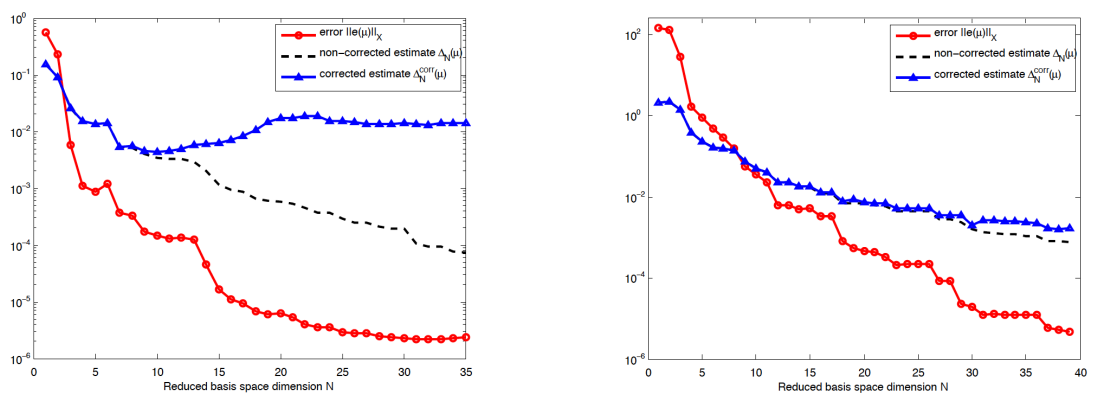

Fig. 2 Convergence of $\sup _{\boldsymbol{\mu} \in \mathcal{D}}\|e(\boldsymbol{\mu})\|_{X}$ versus the corrected $\Delta_{N}^{\text {corr }}(\boldsymbol{\mu})$ and non-corrected $\Delta_{N}(\boldsymbol{\mu})$ error estimates for $M=108$ (left) and $M=208$ (right), respectively.

Including the cost of the offline stage, we estimate that after 500 parametric PDE solutions we have passed the break-even point where RB computations are more efficient. The use of FFD also reduces the number of shape parameters: compared to a local boundary variations approach by moving individual mesh nodes we obtain a reduction of 238:1 in the number of geometric parameters. In Fig. 3 the optimal design for two particular configurations, together with the field solutions, are shown.
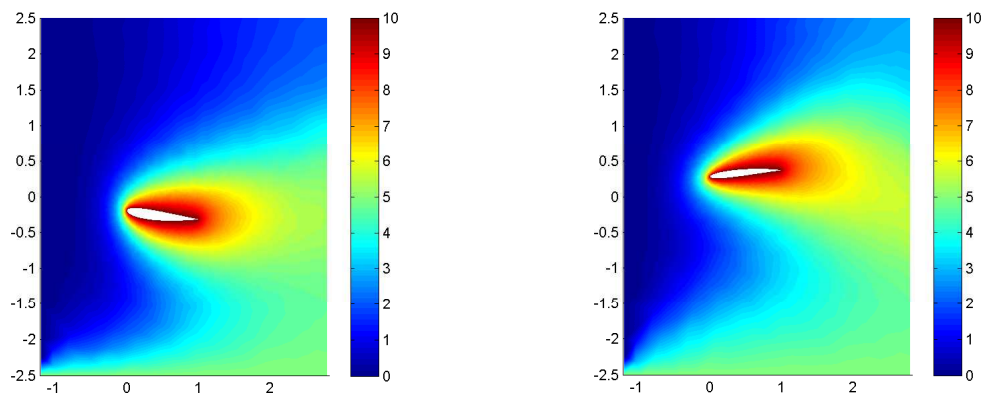

Fig. 3 Optimal design of the airfoil for the cases $\sigma_{0}=7^{\circ}, \bar{u}_{\text {target }}=4.1$ (left) and $\sigma_{0}=$ $-5^{\circ}, \bar{u}_{\text {target }}=4.5$ (right).

\section{Conclusions}

A reduced basis approximation for a shape optimization problem in a thermal flow has been presented. Recovering the assumption of parametric affinity is important to obtain a reduction in the online computational costs. When the geometric transformation map is polynomial, only the diffusive transformation tensor needs to be treated with the empirical interpolation method. This leads to a correction term in the a posteriori error bounds. We have demonstrated that the correction term is rigorous. In the proposed shape optimization problem of an airfoil in thermal flow with four shape parameters 
we observed a reduction of 140:1 in the computational time to solve the RB system vs. the assembling procedure and the solution of the FE system.

Acknowledgements We are grateful to the family of Prof. Fausto Saleri for granting the use of the MLife library as a basis for the numerical FE simulations presented in this paper. We also acknowledge Prof. A. Quarteroni for insights and suggestions and the research group of Prof. Anthony Patera of MIT for the rbMIT [5] package, used for the RB computations.

\section{References}

1. M. Barrault, Y. Maday, N.C. Nguyen, and A.T. Patera. An 'empirical interpolation' method: application to efficient reduced-basis discretization of partial differential equations. C. R. Math. Acad. Sci. Paris, 339(9):667-672, 2004.

2. Y. Chen, J. Hestaven, Y. Maday, and J. Rodriguez. A monotonic evaluation of lower bounds for inf-sup stability constants in the frame of reduced basis approximations. C. R. Acad. Sci. Paris, Ser. I, 346:1295-1300, 2008.

3. L. Dedè. Adaptive and reduced basis methods for optimal control problems in environmental applications. PhD thesis, Politecnico di Milano, 2008.

4. D.B.P. Huynh, D. Knezevic, Y. Chen, J. Hestaven, and A.T. Patera. A natural-norm successive constraint method for inf-sub lower bounds. Submitted, 2009.

5. D.B.P. Huynh, N.C. Nguyen, G. Rozza, and A.T. Patera. Rapid reliable solution of the parametrized partial differential equations of continuum mechanics and transport, 2008. http://augustine.mit.edu.

6. D.B.P Huynh, G. Rozza, S. Sen, and A.T. Patera. A successive constraint linear optimization method for lower bounds of parametric coercivity and inf-sup stability costants. C. R. Acad. Sci. Paris. Sér. I Math., 345:473-478, 2007.

7. T. Lassila and G. Rozza. Parametric free-form shape design with PDE models and reduced basis method. Comput. Meth. Appl. Mech. Engr., in press, 2010.

8. N.C. Nguyen. A posteriori error estimation and basis adaptivity for reduced-basis approximation of nonaffine-parametrized linear elliptic partial differential equations. J. Comp. Phys., 227:983-1006, 2007.

9. A.T. Patera and G. Rozza. Reduced Basis Approximation and A Posteriori Error Estimation for Parametrized Partial Differential Equations. Version 1.0, Copyright MIT 2006, to appear in (tentative rubric) MIT Pappalardo Graduate Monographs in Mechanical Engineering., 2009. Available at http://augustine.mit.edu.

10. A. Quarteroni, G. Rozza, and A. Quaini. Reduced basis methods for optimal control of advection-diffusion problem. In Advances in Numerical Mathematics, W. Fitzgibbon, R. Hoppe, J. Periaux, O. Pironneau, and Y. Vassilevski, Editors, pages 193-216, 2007.

11. A. Quarteroni and A. Valli. Numerical Approximation of Partial Differential Equations. Springer, 2008.

12. G. Rozza. Reduced basis methods for Stokes equations in domains with non-affine parameter dependence. Comput. Vis. Sci., 12(1):23-35, 2009.

13. G. Rozza, D.B.P. Huynh, and A.T. Patera. Reduced basis approximation and a posteriori error estimation for affinely parametrized elliptic coercive partial differential equations. Arch. Comput. Methods Engrg., 15:229-275, 2008.

14. T.W. Sederberg and S.R. Parry. Free-form deformation of solid geometric models. Comput. Graph., 20(4):151-160, 1986.

15. T. Tonn and K. Urban. A reduced-basis method for solving parameter-dependent convection-diffusion problems around rigid bodies. In Proc. ECCOMAS CFD, 2006.

16. K. Veroy, C. Prud'homme, D.V. Rovas, and A.T. Patera. A posteriori error bounds for reduced-basis approximation of parametrized noncoercive and nonlinear elliptic partial differential equations. In Proc. 16th AIAA Comput. Fluid Dynamics, 2003. 